\title{
Turkish Van Cat and Turkish Angora Cat: A Review
}

\author{
Bahattin Cak \\ Department of Animal Science, Faculty of Veterinary Science, University of Yuzuncu Yil, 65080 Van, Turkey
}

\begin{abstract}
Turkish Van and Angora cats are one of most important genetic resources in Turkey. The study aimed to evaluate some morphological properties and also behavioural characteristics in both Turkish Van and Angora cats. This research used both literature and the records from the Van Cat Home at Van Cats Research Centre of Yuzuncu Yil University for Turkish Van cats, as well as literature information for Turkish Angora cat. Van cats which have a special place among world cat races have gained appreciation of people with their original eye colors, long white hair and affectionate attitudes. Origin of Turkish Angora cat breeding in Turkey is Ankara province. Angora cat is strong, energetic, kind, known as a moderately long silky haired cat. According to the results of this study and literature, withers height and body length of Turkish Van cat are higher than the Turkish Angora cat, while tale length and breast girth of Turkish Angora cat are higher than the Turkish Van cat. Body weight (g) of female Turkish Angora cat is heavier than the Turkish Van cat, but male Turkish Van cat is heavier than Turkish Angora cat.
\end{abstract}

Key words: Turkish Van cat, Turkish Angora cat, morphological traits.

\section{Introduction}

Domestic cats entered Anatolia from Eastern Anatolia. According to documents, the Urartians, who lived in the region of Van and their neighbors - the Persians, kept cats and used them in the struggle against mice $[1,2]$. The Turkish Van and Turkish Angora cats are local breeds that were developed in Turkey. The Turkish Van cat was spontaneously originated and should not be confused with the Turkish Angora cat [3]. The Angora cat originates from Ankara (formerly Angora), which is the capital city of Turkey [4]. One of the characteristic features of Van cats is the colour of their eyes. They are classified into three groups according to the colour of their eyes: both eyes blue; both eyes amber (yellow and its tones); one eye blue and the other amber [2]. The Turkish Van cat, which likes swimming, has been reported to exist in the Lake Van region of Eastern Turkey for centuries $[5,6]$. Turkish Angora cat has no relationship between eye color and coat color, and each eye color description can include much variation within its defined spectrum, especially as cats mature.

Corresponding author: Bahattin $\mathrm{Cak}$, assistant professor, research field: animal husbandry.
Acceptable colors include blue, which encompasses shades from sky blue to sapphire; green, which can range from gooseberry to emerald; green-gold, which includes any gold or amber eye that carries a greenish cast or ring; amber, which can range from gold to rich copper but has no green cast or ring; odd-eyed, with one blue eye and the other green, green-gold or amber. While no points are specifically allocated to eye color, deeper, richer tones are preferred. Odd-eyed cats should have similar depth of color in both eyes [7].

It is easy to confuse these two breeds of cat. Blue colour of Van cats usually shows turquoise blue, but amber colour shows many differences in tones. The tones are amber, light amber, yellow and green almond. The classic white Angora might have blue eyes, but a related genetic component frequently renders these cats deaf. If the white Angora has one blue and one yellow eye, it might be deaf in the ear on the same side of the blue eye. The Turkish Van is a bigger cat than the Turkish Angora. The Van also takes longer to mature. They are both affectionate, attractive breeds, but Van cat likes to swim [8,9].

According to the realized study, cat breeds of Turkish Angora and Turkish Van had close relationship with cat breeds of Russian Blue. Those 
Turkish cat breeds also had relationship with cat breeds of British Shorthair/Scottish Fold, Sphynx/Devon Rex. Another study showed that Turkish Angora, Turkish Van and Persian cats were different from Siamese and Bombay cats [10, 11].

Van Yuzuncu Yil University established Van Cat Research Centre in the campus (Figs. 1-3), and the research center had a clinic for cats. Until 2006, about 336 cats are registered and cured by the research center. In 2000s, Van Yuzuncu Yil University and Directorate of Environment of Van Governorship prepared a Van cat project to conserve this breed, but the project could not be continued because of financial difficulties [12].
Ankara Zoo, which belongs to Ministry of Food, Agriculture and Livestock, has a small department to raise Angora cats. They started to be raised in Ankara Zoo since 1939. A study showed that there were some morphological differences between Ankara Zoo Angora cats and Angora cats which are raised at homes in Ankara [4]. Thus, this study aimed to compare the morphological properties and also behavioral characteristic between two different cat breed (Van cats and Angora cats) in Turkey.

\section{Materials and Methods}

The study materials consisted of Van cats in the Van Cat Home at Van Cats Research Centre of

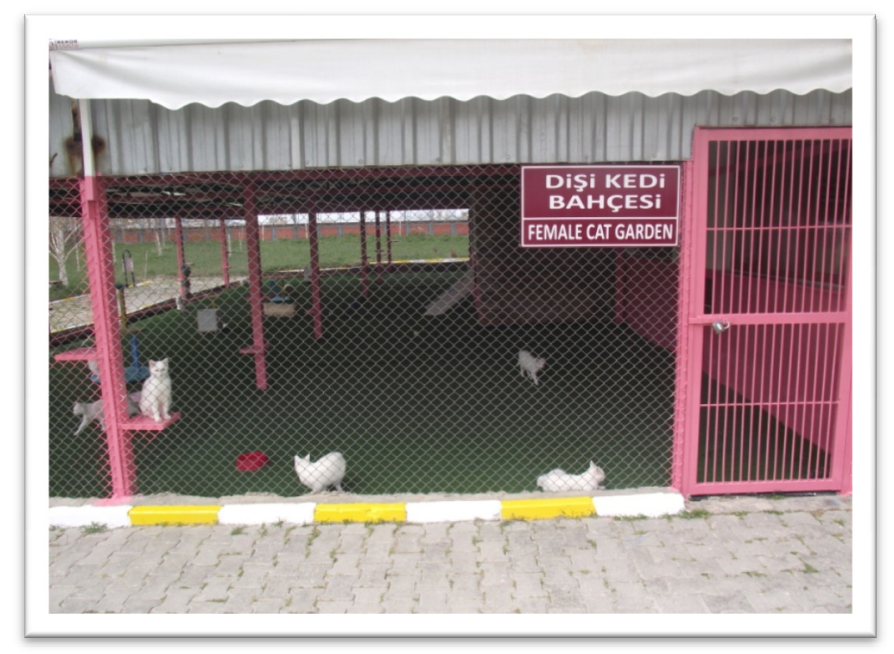

(a) Female cat garden

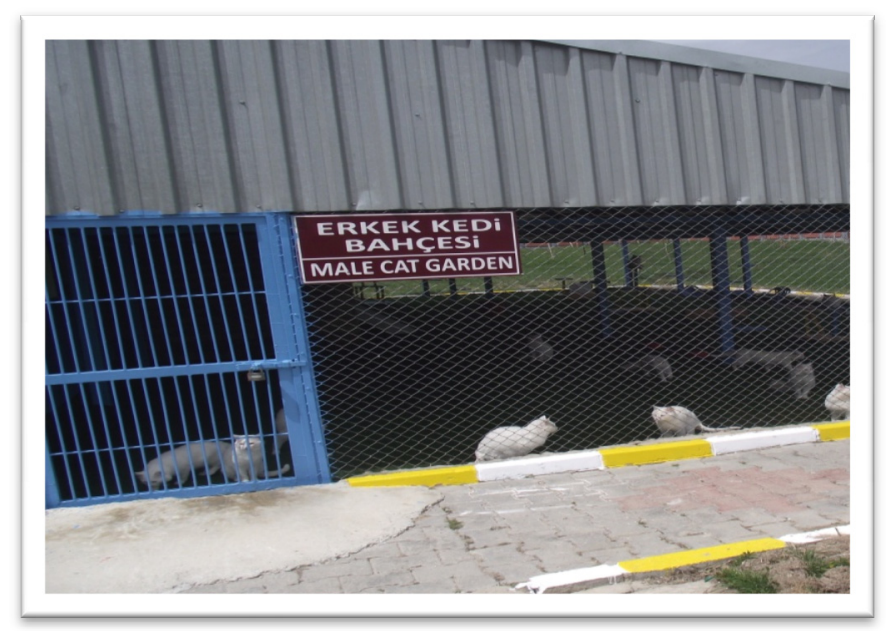

(b) Male cat garden

Fig. 1 Female and male cat garden in Turkish Van Cat Home (photograph by the author). 


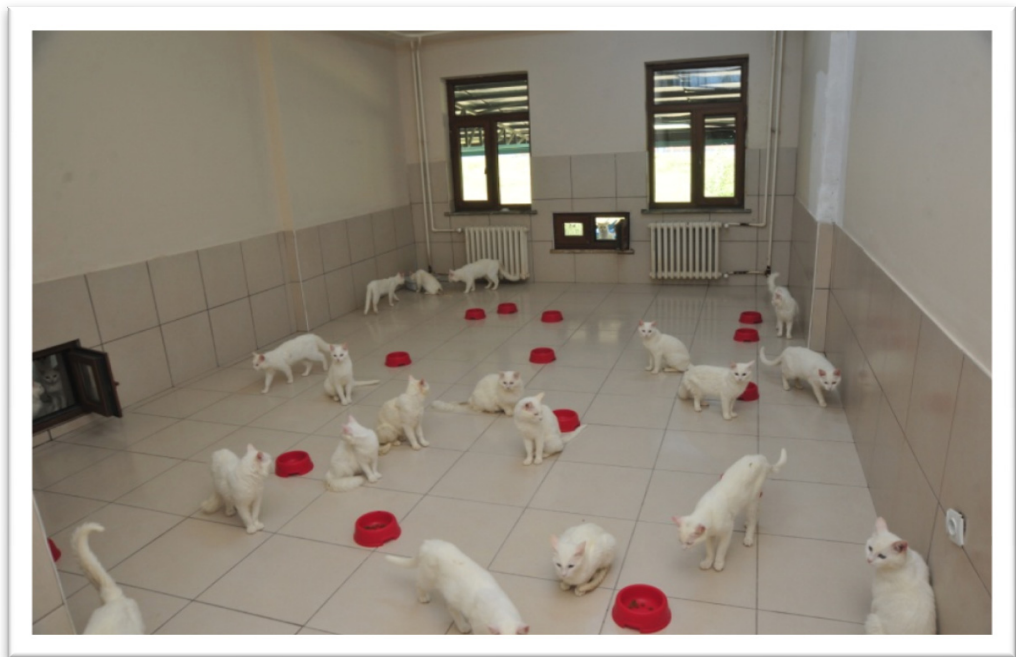

(a) Living room

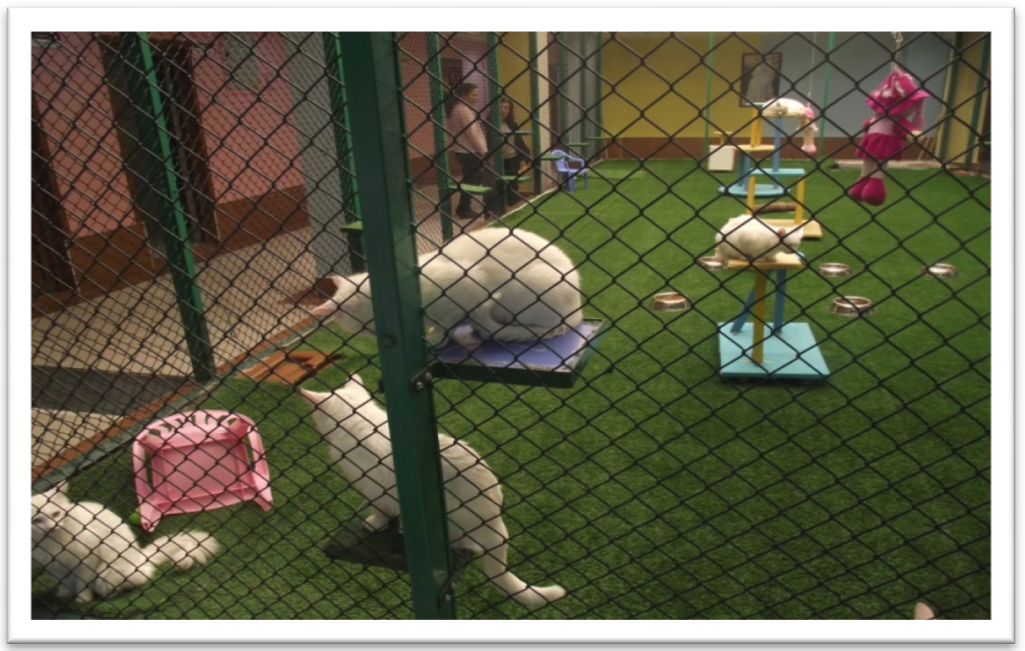

(b) Play room

Fig. 2 Living room and play room in Turkish Van Cat Home (photograph by the author).

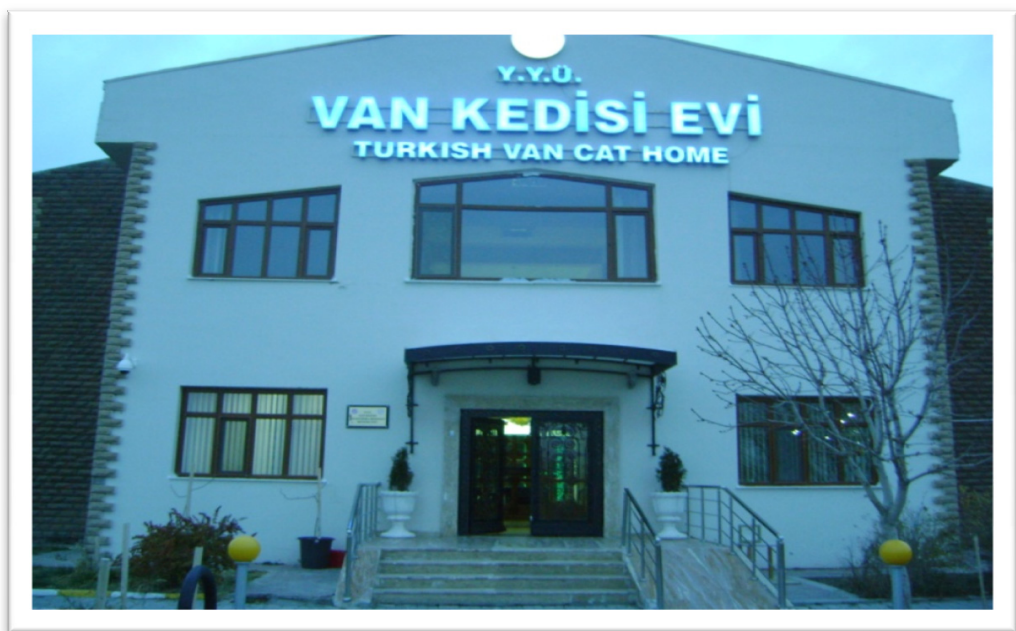

Fig. 3 Turkish Van Cat Home (photograph by the author). 
Yuzuncu Yil University, and the GPS-coordinates of Van are: $38^{\circ} 29^{\prime} 39^{\prime \prime} \mathrm{N}$ and $43^{\circ} 22^{\prime} 48^{\prime \prime} \mathrm{E}$.

This research used both literature and the records of the Van Cats Research Centre. In addition, the study used literature information for Turkish Angora Cat.

\section{Results and Discussion}

This study revealed the definitive values concerning some morphological properties of Turkish Van cat and Turkish Angora cat.

\subsection{Turkish Van Cats}

Some morphological properties of Turkish Van cat are presented in Fig. 4 and Table 1. Turkish Van cats have a middle-size head. There are one or two black points between ears of kittens. Most of the kittens having two or more black spots are odd eye and these black spots are defined almost as stamp of odd eye cats. They are active animals that like to play with water. Cats have three types of hair length as long, medium and short hair. One of the properties that make Van cats attractive is the color of their eyes. They could either have color blue and amber on both eyes, or one blue and one amber eye (odd-eyed) $[2,3$, 13]. They have semi-long with a cashmere-like texture, and soft to the roots with no trace of undercoat. Due to the extremes in climate of their native region, the breed carries two distinctive coat lengths and

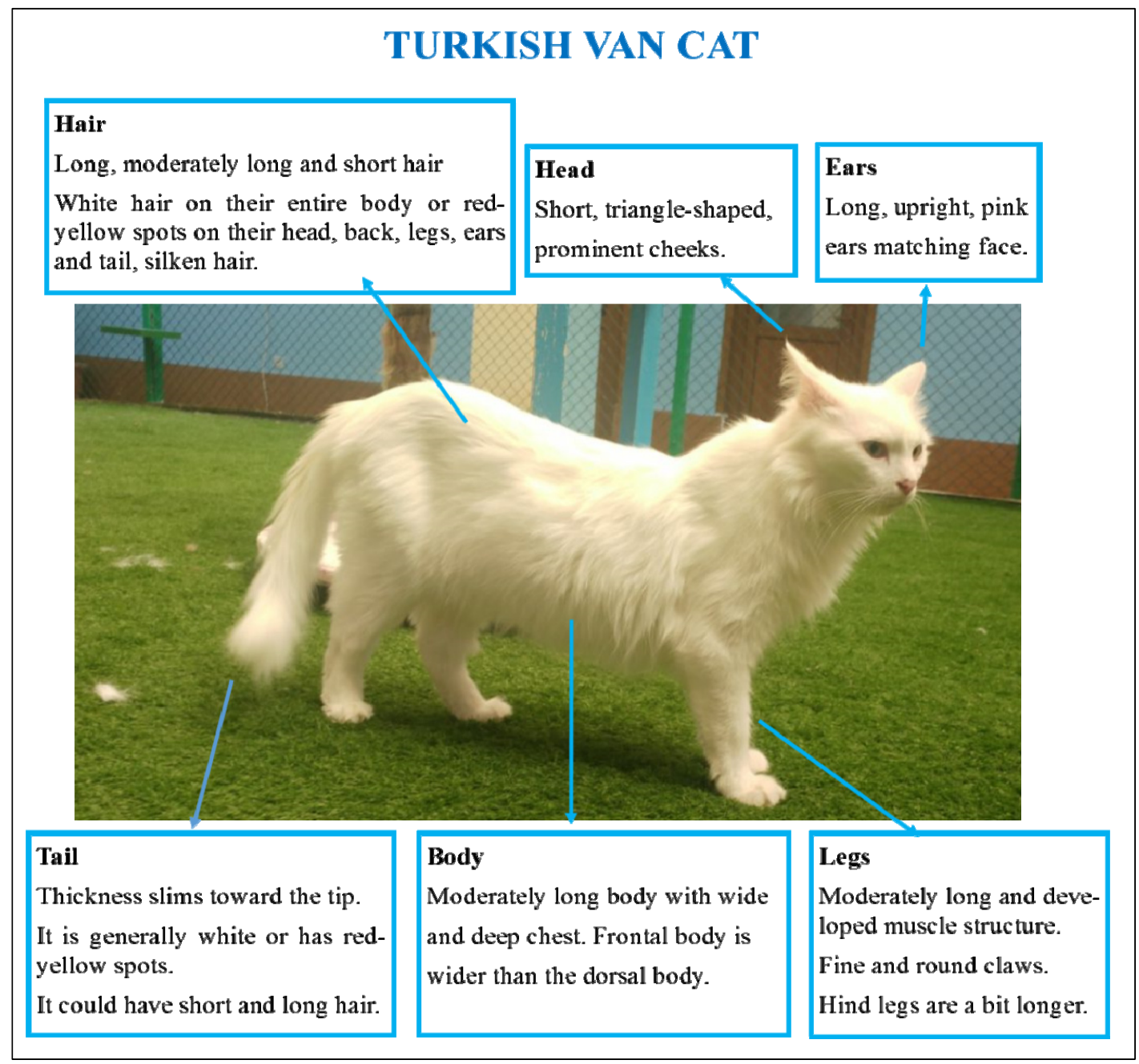

Fig. 4 Some morphological properties of Turkish Van cat (photograph by the author). 
Table 1 Some morphological properties of Turkish Van cat $[2,13,14]$.

\begin{tabular}{ll}
\hline Turkish Van cat & Morphological properties \\
\hline Hair & $\begin{array}{l}\text { Long, moderately long or short hair; white hair on their entire body or red-yellow spots on their head, back, } \\
\text { legs, ears and tail; silken hair }\end{array}$ \\
Head & Short, prominent cheeks \\
Ears & Long, upright, pink ears matching face \\
Legs & Moderately long and developed muscle structure; fine and round claws \\
Body & Moderately long body with wide and deep chest; frontal body is wider than the dorsal body \\
Tail & Thickness slims toward the tip; it is generally white or has red-yellow spots; it could have short and long hair \\
\hline
\end{tabular}

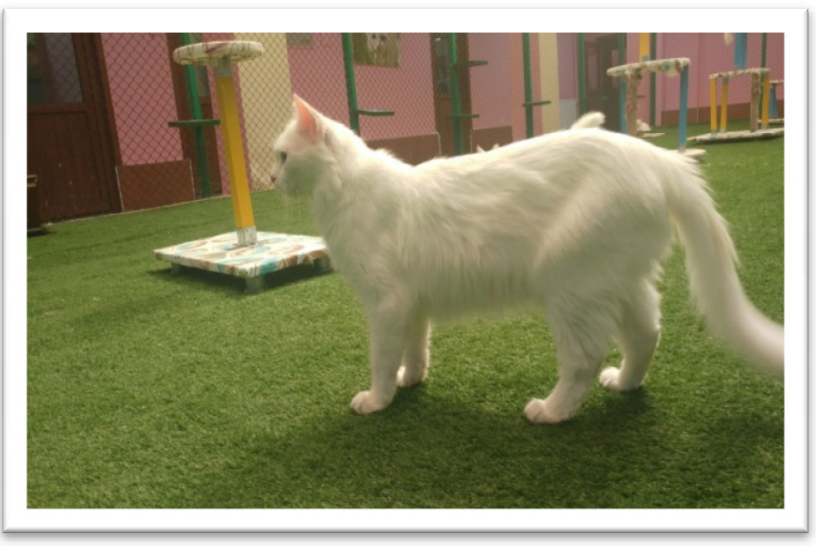

Fig. 5 Turkish Van cat (photograph by the author).

allowances must be made for the seasonal coat. The summer coat is short, conveying the appearance of a shorthair; the winter coat is substantially longer and thicker. There is feathering on the ears, legs, feet and belly. Facial fur is short. A frontal neck ruff and full brush tail become more pronounced with age. The above description is that of an adult, allowances must be made for short coats and tail hair on kittens and young adults [13]. Photographs of Van cats are also given in Fig. 5.

\subsection{Turkish Angora Cats}

Some morphological properties of Turkish Angora cat are presented in Fig. 6 and Table 2. Turkish Angora cat is bred in Ankara province and surrounding area. Harmony between body medium length and body parts is a very good. Feather on the neck and the tail is longer. The tail is long and covered with hairs completely. It is divided in three types according to eye colour. One of the eye may be in blue while the other one is in amber colour, or both may be

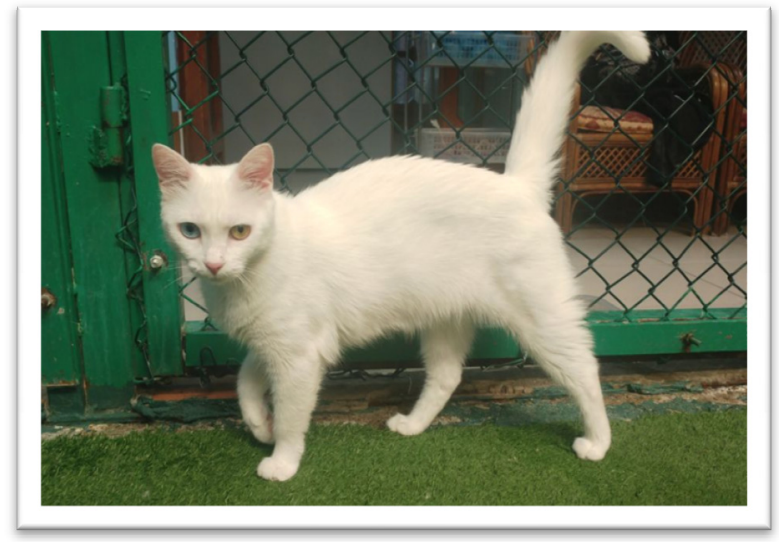

in blue or in yellow colour. Adult animals are seen mane with extension hairs of the winter. Deafness is more common than other breeds [4]. Photographs of Ankara cats are also given in Fig. 7.

\subsection{Morphological Comparison between Turkish Van Cats and Turkish Angora Cats}

\subsubsection{Body Measurements}

Body measurements for Turkish Van cat and Turkish Angora cat are presented in Table 3. Average withers height, body length, tale length and breast girth of male Turkish Van cats are 30, 38, 27 and $30 \mathrm{~cm}$, and for female 27, 36, 26 and $31 \mathrm{~cm}$, respectively. The average body weight for male and female is 3,992 $\mathrm{g}$ and 3,132 $\mathrm{g}$, respectively. While the average withers height, body length, tale length and breast girth are 23, 23, 27, 30 and $39 \mathrm{~cm}$ for male Turkish Angora cats, and 23, 25, 30 and $35 \mathrm{~cm}$ for female Turkish Angora cats. The body weight ( $\mathrm{g}$ ) of Turkish Angora cats are 3,792 $\mathrm{g}$ and 3,359 $\mathrm{g}$ for male and female, respectively. 


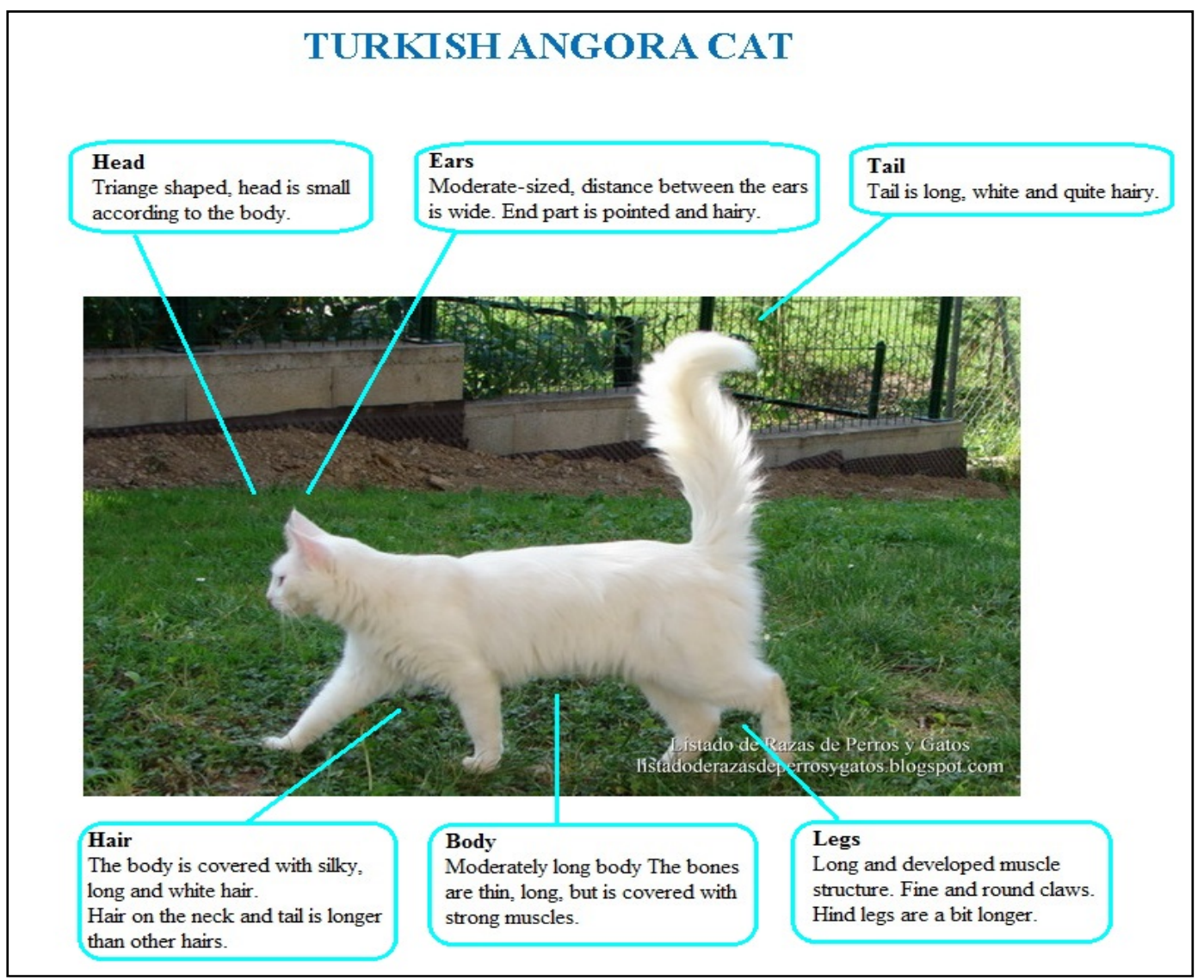

Fig. 6 Some morphological properties of Turkish Angora cat [15].

Table 2 Turkish Angora cat $[4,14]$.

\begin{tabular}{ll}
\hline Turkish Angora cat & Some morphological properties \\
\hline Hair & The body is covered with silky, long, white and shiny hair; hair on the neck and tail is longer \\
Head & Triangle shaped; head is small according to the body \\
Ears & Moderate-sized; distance between the ears is wide; end part is pointed and hairy \\
Legs & Long and developed muscle structure; fine and round claws; hind legs are a bit longer \\
Body & Moderately long body; the bones are thin, long, but is covered with strong muscle \\
Tail & Tail is long, white and quite hairy; it is parallel to the back position in some cases \\
\hline
\end{tabular}
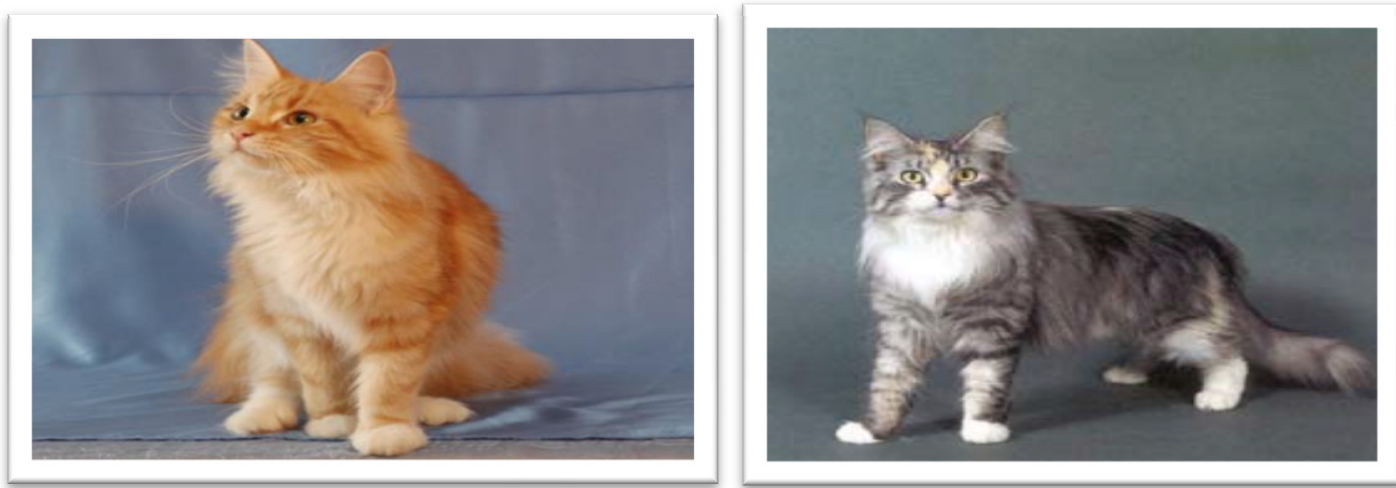

Fig. 7 Examples in various colors of Turkish Angora cat [4]. 
Table 3 Body measurements for the Turkish Van cats $[2,13,14]$ and Turkish Angora cats [4, 14].

\begin{tabular}{|c|c|c|c|c|}
\hline \multirow{2}{*}{ Parameter } & \multicolumn{2}{|c|}{ Turkish Van cat } & \multicolumn{2}{|c|}{ Turkish Angora cat } \\
\hline & Male & Female & Male & Female \\
\hline Withers height $(\mathrm{cm})$ & 31 & 27 & 23 & 22 \\
\hline Body length (cm) & 38 & 36 & 27 & 24 \\
\hline Tale length $(\mathrm{cm})$ & 27 & 25.5 & 30 & 29.5 \\
\hline Breast girth $(\mathrm{cm})$ & 30 & 30.5 & 39 & 34.6 \\
\hline Body weight (g) & 3,992 & 3,132 & 3,792 & 3,359 \\
\hline
\end{tabular}

\subsubsection{Head Structure}

Head structure comparison of Turkish Van and Angora cat is presented in Fig. 8. For Turkish Van cat, head is short, cheeks are protrusive, and ears are long and vertical. While for Turkish Angora cat, head is smaller compared to whole body and triangular shaped. Ears are middle-sized, its bottom is large, its end is pointed vertically, and distance between two ears is long.

\subsubsection{Eyes}

As seen in Fig. 9, eyes of Turkish Van cat are slightly prominent and almond-shaped flattened [3].

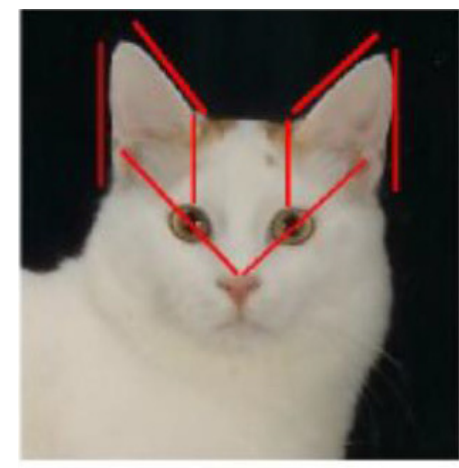

Turkish Van head structure
There are three eye colors: blue, amber and odd-eyed (one blue and one amber) [2]. Eyes are moderately large, a rounded aperture slightly drawn out at the corners, and set at a slant, equidistant from the outside base of the ear to the tip of the nose. Eyes should be clear, alert and expressive [13]. The percentages of odd-eyed, both eyes blue and both eyes amber colour were $26.09 \%, 26.09 \%$ and $47.82 \%$, respectively [2]. Odd eye is called as "tek göz" in Van region. For Turkish Angora cat as seen in Fig. 10, eyes are large, slanting slightly upward with open expression. The acceptable colors include blue, which encompasses

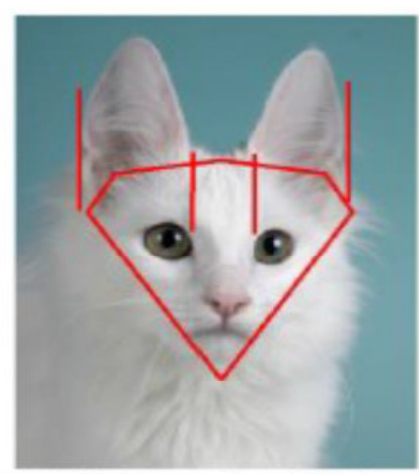

Turkish Ancora head structure

Fig. 8 Head structure comparison between Turkish Van and Angora cat [16].

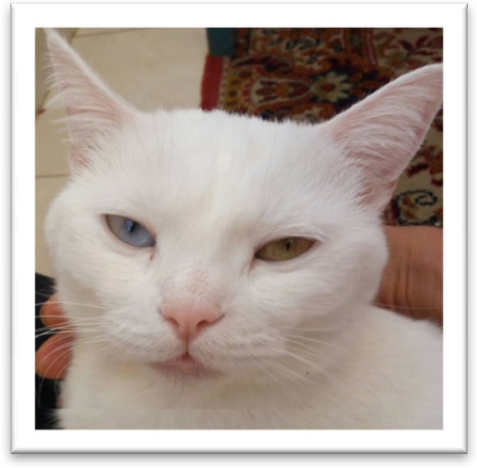

Fig. 9 Eye of Turkish Van cat (photograph by author).

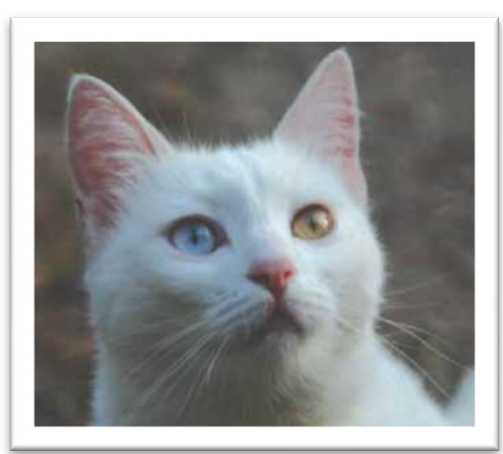

Fig. 10 Eye of Turkish Angora cat [14]. 
shades from sky blue to sapphire; green, which can range from gooseberry to emerald; green-gold, which includes any gold or amber eye that carries a greenish cast or ring; amber, which can range from gold to rich copper, but has no green cast or ring; and odd-eyed, with one blue eye and the other green, green-gold or amber [7]. It was observed that 12 of the 77 cats examined in term of eye colour have odd eye (different eye colour of the same one), the eyes of other 34 cats are yellow and the remained 11 ones have blue eyes [4].

\subsection{Behavioral Characteristics Comparison between Turkish Van Cats and Turkish Angora Cats}

For Turkish Van cat, they always need human attention, and under situation of absence of human attention, they quite become wild. If a member of family fondles a van cat, they jump his/her lap. Van cat is moving, and enjoys being loved and played. It has a high ability to adapt to different environmental conditions. It controls the temperature of the food before eating their meals with their paws. Females are more consistent in their relations with each other. Hunting instinct is developed [14]. The hunting characteristic of Van cats is superior. They hunt rats, mice, bird, flies and insects indoor and outdoor, and eat them. They never attack to poultry animals living with them in the house or out of the house [9]. The females choose males during mating [14]. The cats have more cleaning sense than other animals. They are considerably curious about cleanliness of themselves and their living surrounding. After toilet, they have instinct to burying the dirt. They clean their mouths and faces with help of their paws after eating and toilet [9].

For Turkish Angora cat, it likes to be with family and under its owner interest. It is faithful and close to its owner only. It is nimble, and can express itself well by its voice and body actions. Its reflex against danger is high and it acts fast. Maternal and hunting instincts are very good. The ones in the rural places tend to be independent. It likes to lie on the place where it used to. It is selective about its food $[9,14]$. It keeps its tail horizontal as it is walking, even tail may touch to the head. It is among the clever cat breeds.

For balance of Turkish Van cat, despite age and sex, as adults, individuals should convey an overall impression of a well-balanced and well-proportioned appearance in which no feature is exaggerated to foster weakness or extremes. But, for balance of Turkish Angora cat, proportionate in all physical aspects with a graceful, lithe appearance $[7,13]$.

\section{Conclusions}

According to the results of this study and literature, withers height and body length of Turkish Van cat is higher than the Turkish Angora cat, while tale length and breast girth of Turkish Angora cat is higher than the Turkish Van cat. Body weight (g) of female Turkish Angora cat is heavier than the Turkish Van cat, but male Turkish Van cat is heavier than Turkish Angora cat.

In addition, Turkish Van cat and Turkish Angora cat are affectionately tempered and friendly animals that are regarded as family members and friends with their unprecedented white silken hair, their different eye colours, and their moderately small anatomy. They are among the most important resources of domestic animal genetics in Turkey. They might be breed as special interest and hobby.

\section{Acknowledgments}

Author thanks for contributions of assistant researcher Ahmet Fatih Demirel and Muhammed Oner.

\section{References}

[1] Inan, M. S. 1992. "Biological Distribution of Eye Pigments in Van Cats." Ph.D. thesis, Institute of Science, Van, Turkey.

[2] Ates, C. T. 2000. "Investigation of Morphological and Physiological Properties of the Distribution of Single-Eye Van Cat." Ph.D. thesis, Institute of Health Sciences, Van. 
[3] Odabasioglu, F., and Ates, C. T. 2000. Van Cat. Konya, Turkey: Selcuk University.

[4] Ozcetin, S. T. 2007. "The Morphological, Coat, Growth, Development and Reproduction Characteristics in Angora Cat (Felis catus angorensis)." Ph.D. thesis, Graduate School of Natural and Applied Sciences, Department of Animal Science, Ankara University.

[5] Animal Atlas. 2017. "The Biogeographic Features of Turkey and Turkish Van Cat." Education and Culture Lifelong Learning Programme Comenius. Accessed July 3, 2017. http://www.educa.madrid.org/web/cp.loranca. fuenlabrada/images/Inagenes $\% 20$ comenius/Atlas $\% 20 \mathrm{de}$ \%20animales.pdf.

[6] Fawcett, K. 2016. "Seven Facts about Turkish Van Cats." Accessed June 29, 2017. http://mentalfloss.com/article/83400/7facts-about-turkish van-cat.

[7] The Cat Fanciers' Association. 2014. "Turkish Angora Show Standard (Revised 2014)." The Cat Fanciers' Association, Inc.. Accessed June 6, 2017. http://cfa.org/Portals/0/documents/breeds/standards/turkis h-angora.pdf.

[8] Meggitt, J. 2017. "Difference between Turkish Van and Turkish Angora Cats." Accessed March 3, 2017. http://pets.thenest.com/difference-between-turkish-van-tu rkish-angora-cats-6508.html.

[9] Republic of Turkey Ministry of Culture and Tourism. "Van Cat." Accessed May 26, 2017. https://www.kultur.gov.tr/EN,100044/van-cat.html.

[10] Menotti-Raymond, M., David, V. A., Pflueger, S. M., Lindblad-Toh, K., Wade, C. M., O’Brien, S. J., and Johnson, W. E. 2008. "Patterns of Molecular Genetic
Variation among Cat Breeds.” Journal of Genomics 91 (1): 1-11.

[11] Yilmaz, O., Erturk, Y. E., Coskun, F., Wilson, R. T., and Ertugrul, M. 2016. "Domestic Livestock Resources of Turkey-Cats." Presented at the Symposium of Engineering Science and Nature, Charles University, Prague, Czech Republic.

[12] Avcil, H., Kaptanoglu, M., and Akova, R. 2006. "Environmental Status Report of Van Province." Directorate of Environment and Forestry, Province of Van. Accessed May 25, 2017. http://www.vanherbaryum.yyu.edu.tr/vanre/vilcdr.pdf.

[13] The Cat Fanciers' Association. 2009. "Turkish Van Show Standard (Revised 2009).” The Cat Fanciers' Association, Inc.. Accessed June 6, 2017. http://cfa.org/Portals/0/documents/breeds/standards/turkis h-van.pdf.

[14] Ministry of Food Agriculture and Livestock. 2011. "Domestic Animal Genetic Resources in Turkey." General Directorate of Agricultural Research and Policy. Accessed December 2011. https://www.rfpeurope.org/fileadmin/SITE_ERFP/countr y_reports/Turkey/TU_AnGR.pdf.

[15] Blogspot. 2013. "Angora Cat." List of Breeds of Dogs and Cats. Accessed June 5, 2017. http://listadoderazasdeperrosygatos.blogspot.com.tr/2013/ 02/raza-angora-turco.html.

[16] The Cat Fanciers' Association. 2016. "Turkish Vans." Judges Workshop 2016 Annual Meeting. Accessed March 03, 2017. http://cfa.org/Portals/0/documents/judges-ce/TurkishVan. pdf. 